\title{
FOREWORD
}

\section{Special feature on imaging systems and techniques}

\section{Guest Editors}

\author{
Wuqiang Yang \\ University of Manchester, \\ $U K$
}

\section{George Giakos \\ University of Akron, $\mathrm{OH}$, USA}

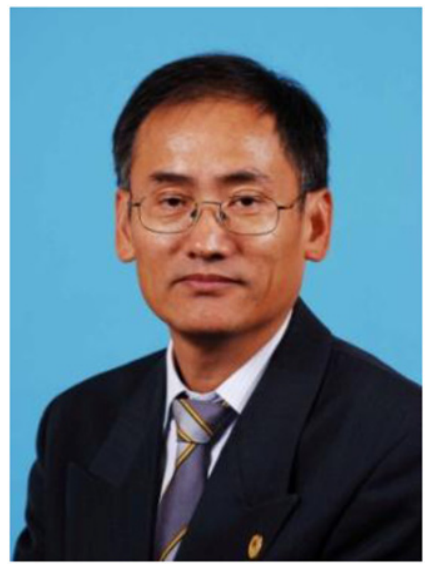

Wuqiang Yang

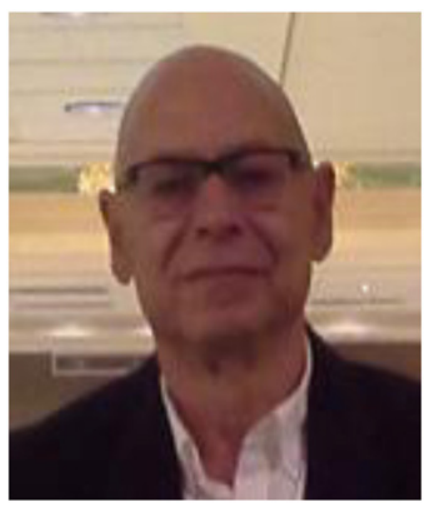

George Giakos
The IEEE International Conference on Imaging Systems and Techniques (IST'2012) was held in Manchester, UK, on 16-17 July 2012. The participants came from 26 countries or regions: Austria, Brazil, Canada, China, Denmark, France, Germany, Greece, India, Iran, Iraq, Italy, Japan, Korea, Latvia, Malaysia, Norway, Poland, Portugal, Sweden, Switzerland, Taiwan, Tunisia, UAE, UK and USA. The technical program of the conference consisted of a series of scientific and technical sessions, exploring physical principles, engineering and applications of new imaging systems and techniques, as reflected by the diversity of the submitted papers. Following a rigorous review process, a total of 123 papers were accepted, and they were organized into 30 oral presentation sessions and a poster session. In addition, six invited keynotes were arranged. The conference not only provided the participants with a unique opportunity to exchange ideas and disseminate research outcomes but also paved a way to establish global collaboration.

Following the IST'2012, a total of 55 papers, which were technically extended substantially from their versions in the conference proceeding, were submitted as regular papers to this special feature of Measurement Science and Technology. Following a rigorous reviewing process, 25 papers have been finally accepted for publication in this special feature and they are organized into three categories: (1) industrial tomography, (2) imaging systems and techniques and (3) image processing. These papers not only present the latest developments in the field of imaging systems and techniques but also offer potential solutions to existing problems. We hope that this special feature provides a good reference for researchers who are active in the field and will serve as a catalyst to trigger further research.

It has been our great pleasure to be the guest editors of this special feature. We would like to thank the authors for their contributions, without which it would not be possible to have this special feature published. We are grateful to all reviewers, who devoted their time and effort, on a voluntary basis, to ensure that all submissions were reviewed rigorously and fairly. The publishing staff of Measurement Science and Technology are particularly acknowledged for giving us timely advice on guest-editing this special feature. 